UDC 519.688, 539.126

DOI: $10.22363 / 2658-4670-2020-28-3-216-229$

\title{
Application of a computer algebra systems to the calculation of the $\pi \pi$-scattering amplitude
}

\author{
Yu. L. Kalinovsky ${ }^{1,2}$, A. V. Friesen ${ }^{1}$, \\ E. D. Rogozhina ${ }^{1,2}$, L. I. Golyatkina ${ }^{1,2}$ \\ ${ }^{1}$ Joint Institute for Nuclear Research \\ 6, Joliot-Curie St., Dubna, Moscow region, 141980, Russian Federation \\ ${ }^{2}$ Dubna State University \\ 19, Universitetskaya St., Dubna, Moscow Region, 141982, Russian Federation
}

(received: July 6, 2020; accepted: September 14, 2020)

The aim of this work is to develop a set of programs for calculation the scattering amplitudes of the elementary particles, as well as automating the calculation of amplitudes using the appropriate computer algebra systems (Mathematica, Form, Cadabra). The paper considers the process of pion-pion scattering in the framework of the effective Nambu-Iona-Lasinio model with two quark flavours. The Package$X$ for Mathematica is used to calculate the scattering amplitude (starting with the calculation of Feynman diagrams and ending with the calculation of Feynman integrals in the one-loop approximation). The loop integrals are calculated in general kinematics in Package- $X$ using the Feynman parametrization technique. A simple check of the program is made: for the case with zero temperature, the scattering lengths $a_{0}=0.147$ and $a_{2}=-0.0475$ are calculated and the total cross section is constructed. The results are compared with other models as well as with experimental data.

Key words and phrases: Feynman integrals, one-loop approximation, total sross section, scattering length, a computer algebra, Package-X

\section{Introduction}

The heavy ion collision experiment is an instrument for the study of the matter properties under critical conditions. The modern experiment is a multistage process, which includes the event selection, the event reconstruction (the reconstruction of the primary particles) and the simulation of the collision process. The simulation is made on the base of the chosen model and the final result has to reproduce the real data. The fulfil of such analysis or simulation among other things requires a good understanding and a strict description of the final state particle interaction.

The information about the particles properties and their interactions can be extracted from the probabilities of the processes occurring during their

(C) Kalinovsky Y.L., Friesen A. V., Rogozhina E. D., Golyatkina L. I., 2020

This work is licensed under a Creative Commons Attribution 4.0 International License

http://creativecommons.org/licenses/by/4.0/ 
collision. The interaction probability is associated with the cross section of the given reaction and the phase volume, which is uniquely determined by the laws of conservation of energy-momentum, i.e., by the kinematics of the reaction. From the theoretical point of view, the cross section is defined by the scattering amplitude, which is described in the framework of the model under consideration. The model can include description of the quantum mechanical properties of the particles, describe the type of the interaction, take into account the matter properties or the quark structure of the colliding particles, etc. That is why to obtain the scattering amplitude is not the trivial task both from theoretical and computing point of view.

This paper is dedicated to the calculation of $\pi \pi$-scattering amplitude. As the lightest hadron with Goldstone nature, the pion occupies a special position in hadronic physics. The elastic $\pi \pi$-scattering is a fundamental process for quantum chromodynamics (QCD) at low energies as it provides an ideal testing for the mechanism of spontaneous chiral symmetry breaking. During the heavy ion collision, pions can be quickly created in the early phase, for example, by the Schwinger mechanism and their number is quasi-conserved over the time scale of the heavy ion collision until freeze- out. Then, in the hadron phase, two and more pions are the final state of many hadronic interactions. Accordingly, $\pi \pi-$ scattering attracts considerable interest even though the cross section is not directly measurable.

In this work the $\pi \pi$-scattering is considered in the frame of two-flavour Nambu-Jona-Lasinio model (NJL) [1]. The most important advantage of the NJL model is that it introduces a mechanism of the dynamical breaking of chiral symmetry (due to the quark-antiquark condensate). The model has a good tool to introduce finite temperature suggested by Matsubara and is able to describe the matter properties at finite temperature and density. Nevertheless, the calculation of the $\pi \pi$-scattering amplitude in NJL model is difficult due to the appearing of four-point one-loop Feynman integrals, that make the using of Matsubara mechanism in the general kinematics complicated. That is why the problem was solved only for limiting cases, for example, for the case with $p_{1}=p_{2}=p_{3}=p_{4}=p$ [2]. This limit allows to estimate the scattering lengths $a_{0}, a_{2}$, but does not make it possible to evaluate the cross section, the pion damping width or the lifetime. This work focuses on the evaluation of the scattering amplitude in general kinematics but due to the use of the dimensional regularization scheme, without the finite temperature.

The evaluation of the scattering amplitude can be made in two steps: writing down the amplitude using the Feynman rules, evaluating traces and then integrating over the phase space, applying the chosen kinematics. Many packages allow to evaluate Dirac traces, for example, Form [3] or Cadabra [4]. Obtained results usually require a lot of additional analytical work for writing down the amplitude in appropriate form and for numerical calculation. To evaluate one-loop integrals that appear in higher order calculations of perturbative quantum field theory, there are exist, for example, the Mathematica packages FeynCalc [5], FormCalc [6] and the Fortran program Golem95 [7]. These packages compute the one-loop tensor integrals using the Passarino-Veltman reduction algorithm [8]. In this work we use the Package-X for Mathematica, developed by Hiren H. Patel [9]. The Package-X runs all steps for amplitude evaluation: performing traces over products of 
Dirac matrices, giving the result in the term of Passarino-Veltman functions, computation of the one-loop integrals. Package-X provides analytic expressions for UV-divergent, IR-divergent and finite parts either separately or all together. Output expressions can be readily evaluated numerically and manipulated symbolically with built-in Mathematica functions.

\section{Using the Package-X for the evaluation of $\pi-\pi$ scattering amplitude}

In this work the meson-meson scattering amplitude in the frame of effective QCD models is considered. The work focuses on the NJL model [1], the Lagrangian of the model can consist of scalar, pseudo-scalar, vector, and axial-vector four-quark interactions. The expressions for the total amplitude $\mathcal{T}(s, t, u)$ depend on the number of interactions taken into account. For this work the Lagrangian with two flavours of quarks and scalar and pseudo-scalar four-quark interactions is chosen:

$$
\mathcal{L}_{\mathrm{NJL}}=\bar{q}\left(i \gamma_{\mu} \partial^{\mu}-\hat{m}_{0}\right) q+G\left[(\bar{q} q)^{2}+\left(\bar{q} i \gamma_{5} \vec{\tau} q\right)^{2}\right],
$$

where $G$ is the scalar coupling constant, $\bar{q}, q$ are the quark fields, $\hat{m}_{0}$ is the diagonal matrix of the current quark mass, $\hat{m}_{0}=\operatorname{diag}\left(m_{u}^{0}, m_{d}^{0}\right)$ with $m_{u}^{0}=m_{d}^{0}=m_{0}$, and $\vec{\tau}$ are the Pauli matrices in space $\mathrm{SU}(2), \tau^{a}(a=1,2,3)$.

In the mean field approximation the constituent mass of the quark is provided by the gap equation:

$$
m=m_{0}+2 i G \int \frac{d p}{(2 \pi)^{4}} \operatorname{Tr}\{S(p)\},
$$

where $S(p)=(\hat{p}-m)^{-1}$ is the quark propagator and trace is taken over Dirac, flavour and colour indexes.

A meson is considered as a quark-antiquark couple forming a loop, and in the framework of the RPA approximation it leads to the Bethe-Salpeter equation:

$$
1-\left.2 G \Pi_{M}\left(k^{2}\right)\right|_{k^{2}=M^{2}}=0,
$$

with the polarization operator $\Pi_{M}\left(k^{2}\right)$, which defines the meson properties:

$$
\Pi_{M}\left(k^{2}\right)=i \int \frac{d^{4} p}{(2 \pi)^{4}} \operatorname{Tr}\left[\Gamma_{M} S(p+k) \Gamma_{M} S(p)\right],
$$

where the vertex factor $\Gamma_{M}$ depends on the sort of meson. $\Gamma_{M}=i \gamma_{5} \tau^{a}$ for the pseudo-scalar meson and $\Gamma_{M}=\mathbf{1} \tau^{a}$ for scalar meson. The meson propagator is defined as

$$
D_{M}\left(k^{2}\right)=\frac{2 i G}{1-2 G \Pi_{M}\left(k^{2}\right)} .
$$

For simplicity, in this work the parametrization suggested in the work [10] is used. The set of equations for fixing masses and coupling constants can be written as: 


$$
M_{s}^{2}=M_{\pi}^{2} Z+4 m^{2}, \quad g_{\sigma q q}=g_{\pi q q} \sqrt{Z}
$$

where $Z=1-6 m^{2} / M_{a_{1}}^{2}, M_{a_{1}}=1.2 \mathrm{GeV}$ is the mass of $a_{1}$-meson, $M_{\pi}=0.14$ $\mathrm{GeV}$ is the pion mass and $m$ is the constituent quark mass. The coupling constant $g_{\pi q q}$ can be obtained from the Goldberger-Treiman relation [11]:

$$
g_{\pi q q} f_{\pi}=m+\mathcal{O}(m),
$$

where $f_{\pi}=0.0924 \mathrm{GeV}$ is the pion decay constant.

On the lowest order $1 / N_{c}$ there are two types of Feynman diagrams, contributed to the $\pi \pi$-scattering amplitude: four-vertex "box "-diagrams and the meson-exchange diagrams [2], [12]. The set of "box"-diagram is presented in the Figure 1 . Here $p_{1}, p_{2}$ are the momenta of incoming particles, $p_{3}, p_{4}$ are the momenta of scattered particles, $k$ is the integration variable.

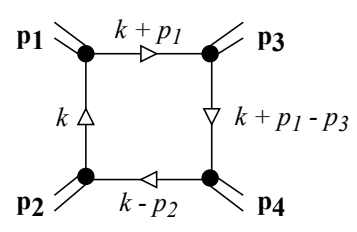

a)

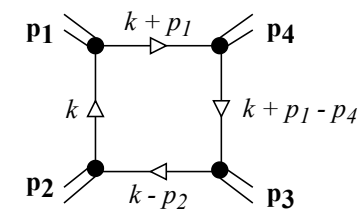

b)

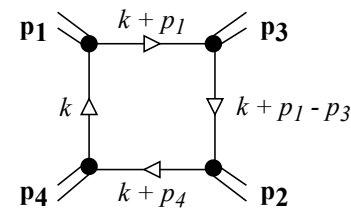

c)

Figure 1. Feynman diagrams contributing to the $\pi \pi$-scattering

The amplitudes corresponding to each diagram in Figure 1 can be written as:

$$
i \mathcal{T}=-g_{\pi q q}^{4} \mu^{2 \epsilon} \int \frac{d^{d} k}{(2 \pi)^{d}} \frac{\operatorname{Tr}\left\{\gamma_{5}\left(\hat{q_{1}}+m\right) \gamma_{5}\left(\widehat{q_{2}}+m\right) \gamma_{5}\left(\widehat{q_{3}}+m\right) \gamma_{5}\left(\widehat{q_{4}}+m\right)\right\}}{\left[q_{1}^{2}-m^{2}\right]\left[q_{2}^{2}-m^{2}\right]\left[q_{3}^{2}-m^{2}\right]\left[q_{4}{ }^{2}-m^{2}\right]} .
$$

The prefactor $-g_{\pi q q}^{4}$ appears as the vertex factor in the diagrams are $\Gamma_{\pi}=i g_{\pi q q} \gamma_{5}$. The notation $q_{i}, i=1, \ldots, 4$ was introduced for the simplicity and the values of $q_{i}$, corresponding to diagrams in Figure $1 \mathrm{a}$ ), b) c) accordingly are listed in Table 1.

The $q_{i}$ for the "box"-diagrams in Figure 1

Table 1

\begin{tabular}{|l|l|l|l|}
\hline & $T_{s}^{b o x}(\mathrm{a})$ & $T_{u}^{b o x}(\mathrm{~b})$ & $T_{t}^{b o x}(\mathrm{c})$ \\
\hline$q_{1}$ & $k$ & $k$ & $k$ \\
$q_{2}$ & $k-p_{2}$ & $k-p_{2}$ & $k+p_{4}$ \\
$q_{3}$ & $k+p_{1}-p_{3}$ & $k+p_{1}-p_{4}$ & $k+p_{1}-p_{3}$ \\
$q_{4}$ & $k+p_{1}$ & $k+p_{1}$ & $k+p_{1}$ \\
\hline
\end{tabular}

To compute traces of Dirac matrices in denominator of equation 7 the function Spur is used. The LoopIntegrate function initiates the evaluation 
of integral and carries out its covariant tensor decomposition in term of Passarino-Veltman functions (PVA, PVB, PVC, PVD). For diagrams in Figure 1, keeping the notation for s-, t-, u- channels, it should be written:

$\operatorname{In}[2]:=$

$\mathrm{sChannel}=$ LoopIntegrate $\left[\operatorname{Spur}\left[\gamma \cdot \mathrm{k}+m_{1}, \gamma_{5}, \gamma \cdot\left(\mathrm{k}-p_{2}\right)+m_{1}\right], \gamma_{5}, \gamma \cdot\left(\mathrm{k}+p_{1}-p_{3}\right)+\right.$ $\left.\left.m_{1}, \gamma_{5}, \gamma \cdot\left(\mathrm{k}+p_{1}\right)+m_{1}, \gamma_{5}\right], \mathrm{k},\{\mathrm{k}, \mathrm{m}\},\left\{\mathrm{k}-p_{2}, \mathrm{~m}\right\},\left\{\mathrm{k}+\left(p_{1}-p_{3}\right), \mathrm{m}\right\},\left\{\mathrm{k}+p_{1}, \mathrm{~m}\right\}\right] ;$ uChannel $=$ LoopIntegrate[Spur $\left[\gamma \cdot \mathrm{k}+m_{1}, \gamma_{5}, \gamma \cdot\left(\mathrm{k}-p_{2}\right)+m_{1}\right], \gamma_{5}, \gamma \cdot\left(\mathrm{k}+p_{1}-p_{4}\right)+$ $\left.\left.m_{1}, \gamma_{5}, \gamma \cdot\left(\mathrm{k}+p_{1}\right)+m_{1}, \gamma_{5}\right], \mathrm{k},\{\mathrm{k}, \mathrm{m}\},\left\{\mathrm{k}-p_{2}, \mathrm{~m}\right\},\left\{\mathrm{k}+p_{1}-p_{4}, \mathrm{~m}\right\},\left\{\mathrm{k}+p_{1}, \mathrm{~m}\right\}\right] ;$ tChannel $=$ LoopIntegrate[Spur $\left[\gamma \cdot \mathrm{k}+m_{1}, \gamma_{5}, \gamma \cdot\left(\mathrm{k}+p_{1}+p_{2}-p_{3}\right)+m_{1}\right], \gamma_{5}, \gamma \cdot\left(\mathrm{k}+p_{1}\right.$ $\left.\left.-p_{3}\right)+m_{1}, \gamma_{5}, \gamma \cdot\left(\mathrm{k}+p_{1}\right)+m_{1}, \gamma_{5}\right], \mathrm{k},\{\mathrm{k}, \mathrm{m}\},\left\{\mathrm{k}+p_{1}+p_{2}-p_{3}, \mathrm{~m}\right\},\left\{\mathrm{k}+p_{1}-p_{3}\right.$, $\left.\mathrm{m}\},\left\{\mathrm{k}+p_{1}, \mathrm{~m}\right\}\right]$

The output of the LoopIntegrate function contains a number of dotproducts of the external momenta. It is possible to define on-shell conditions expressing scalar products in terms of Mandelstam invariants with the function MandelstamRelations and eliminate one of variables using additional command Eliminate.

$\operatorname{In}[1]:=$

onShell $=$ MandelstamRelations $\left[p_{1}, p_{2}, p_{3}, p_{4}, \mathrm{M}, \mathrm{M}, \mathrm{M}, \mathrm{M} \rightarrow \mathrm{s}, \mathrm{t}, \mathrm{u}\right.$, Eliminate $\left.\rightarrow \mathrm{u}\right]$

Out[1]:=

$\left\{\mathrm{p} 1^{2} \rightarrow M^{2}, \mathrm{p} 2^{2} \rightarrow M^{2}, \mathrm{p} 3^{2} \rightarrow M^{2}, \mathrm{p} 4^{2} \rightarrow M^{2}, \mathrm{p} 1 . \mathrm{p} 2 \rightarrow \frac{1}{2}\left(s-2 M^{2}\right), \mathrm{p} 3 . \mathrm{p} 4 \rightarrow\right.$

$\frac{1}{2}\left(s-2 M^{2}\right), \mathrm{p} 1 . \mathrm{p} 3 \rightarrow \frac{1}{2}\left(2 M^{2}-t\right), \mathrm{p} 2 \cdot \mathrm{p} 4 \rightarrow \frac{1}{2}\left(2 M^{2}-t\right), \mathrm{p} 1 . \mathrm{p} 4 \rightarrow$

\begin{tabular}{l}
$\left.\frac{1}{2}\left(-2 M^{2}+s+t\right), \mathrm{p} 2 \cdot \mathrm{p} 3 \rightarrow \frac{1}{2}\left(-2 M^{2}+s+t\right), \varepsilon^{\{\mathrm{p} 1\}\{\mathrm{p} 2\}\{\mathrm{p} 3\}\{\mathrm{p} 4\}} \rightarrow 0\right\}$ \\
\hline
\end{tabular}

After covariant decomposition and on-shell or kinematic conditions are applied, the result in term of the coefficient functions is obtained. At the final step LoopRefine is run to reduce the obtained result in terms of analytic expressions DiscB, ScalarC0, ScalarD0, that are the in-built Mathematica functions and are suitable for numerical calculation. Actually, these functions are an abbreviation for a more complicated expression and the function DiscExpand displays them in terms of elementary functions.

\section{$\operatorname{In}[5]:=$}

sChannelInt $=$ LoopRefine[sChannel $/$. onShell]

uChannelInt $=$ LoopRefine[tChannel $/$. onShell];

tChannelInt $=$ LoopRefine[uChannel $/$. onShell];

Out[5]:=

$8+4\left(\frac{1}{\epsilon}+\log \left[\frac{2}{\mathrm{~m}^{2}}\right]\right)+2 \operatorname{DiscB}[\mathrm{s}, \mathrm{m}, \mathrm{m}]+2 \operatorname{DiscB}[\mathrm{t}, \mathrm{m}, \mathrm{m}]-$

$2\left(2 M^{2}-s\right)$ ScalarC0 $\left[\mathrm{M}^{2}, \mathrm{M}^{2}, \mathrm{~s}, \mathrm{~m}, \mathrm{~m}, \mathrm{~m}\right]-2\left(2 M^{2}-t\right)$ ScalarC0 $\left[\mathrm{M}^{2}, \mathrm{M}^{2}, \mathrm{t} ; \mathrm{m}, \mathrm{m}, \mathrm{m}\right]+$ $\left(2 \mathrm{M}^{4}-\mathrm{st}\right)$ ScalarD0 $\left[\mathrm{M}^{2}, \mathrm{M}^{2}, \mathrm{M}^{2}, \mathrm{M}^{2} ; \mathrm{s}, \mathrm{t} ; \mathrm{m}, \mathrm{m}, \mathrm{m}, \mathrm{m}\right]$

There are a few notes concerning the outputs: in the outputs an overall factor $i /\left(16 \pi^{2}\right)$ is omitted for brevity, and should be restored during analysis of the result. The result can contain the term $1 / \epsilon$ that always explicitly displays logarithmic UV and/or IR divergences. During the analysis of the 
results, it should be kept in mind that the term $1 / \epsilon$ is equal to $(1 / \epsilon-\gamma+\ln 4 \pi)$. The discussion about the renormalization in QED and QCD can be found, for example, in the work [13]. The UV and/or IR divergence can be displayed separately using Part $\rightarrow$ UVDivergent.

\section{$\operatorname{In}[8]:=$}

LoopRefine[sChannel, Part $\rightarrow$ UVDivergent]

Out $[8]:=\frac{1}{4 \epsilon \pi^{2}}$

The scattering amplitude, expressed in variable $s, t, u$, is invariant and does not change when replacing $s \leftrightarrow t, s \leftrightarrow u, t \leftrightarrow u$. Therefore, the first diagram in Figure 1 a) can be obtained from the second one b) by replacing $t \leftrightarrow u$, and the third c) from the second b) - by replacing $s \leftrightarrow t$. Using this property, the simple check of result can be done:

\section{$\operatorname{In}[9]:=$}

sChannelf $\left[\mathrm{s}_{-}, \mathrm{t}_{-}, \mathrm{u}_{-}\right]=\mathrm{sChannel} /$. onShell;

uChannelf $\left[s_{-}, \bar{t}_{-}, \bar{u}_{-}\right]=$uChannel $/$. onShell;

tChannelf $\left[s_{-}, t_{-}, u_{-}^{-}\right]=$tChannel $/$. onShell;

FullSimplify[LoopRefine[sChannelf[s, t, u] - uChannelf[s, u, t]]]

FullSimplify[LoopRefine[uChannelf[s, t, u] - tChannelf[t, s, u]]]

Out [12]: $=0$

Out $[13]:=0$
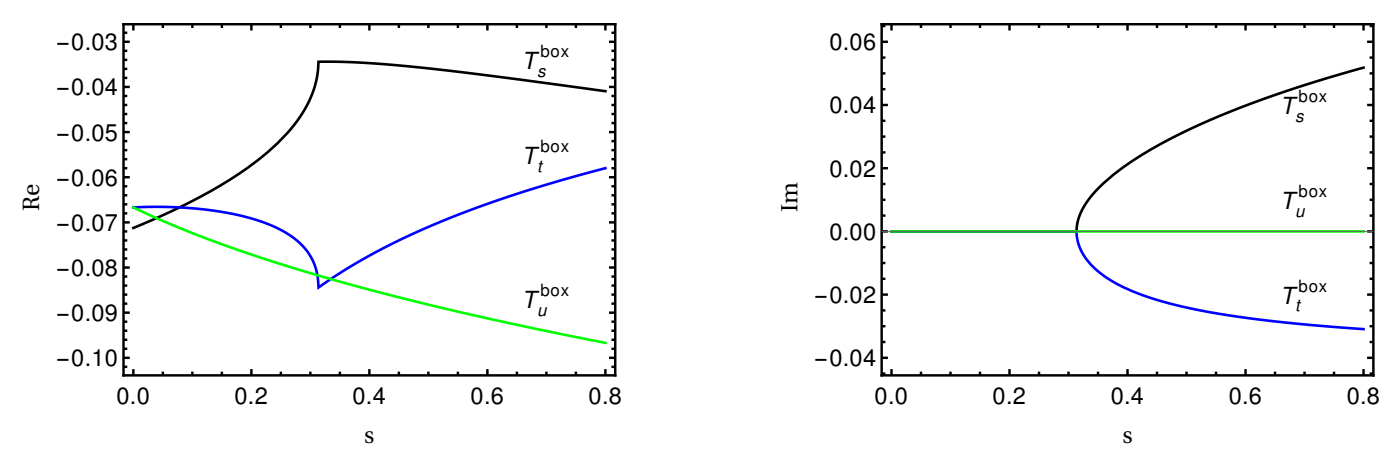

Figure 2. The real (left panel) and imaginary (right panel) of the "box"-diagrams for the case $t=0$

The real and imaginary parts of the integral (7) for all "box"-diagrams as function of $s$ are shown in Figure 2 for the case $t=0$.

The second type of Feynman diagrams contributed to the $\pi \pi$-scattering amplitude is the diagram with the meson as intermediate state. As the intermediate state there can be scalar, vector, axial-vector mesons if they appear in the Lagrangian of the model. The diagrams with intermediate meson propagator are shown in Figure 3, where triangle vertexes correspond to $M \rightarrow \pi \pi$ decay and d) e) and f) will be denoted as s-, u-, t- channels accordingly. According to the Lagrangian (1), in the model only scalar and 
pseudo-scalar quark-antiquark states are present and as the intermediate state the scalar $\sigma$-meson appears. Accordingly, for this case, the triangle vertexes correspond to $\sigma \rightarrow \pi \pi$ decay. The total amplitude for the meson-exchange diagram is written as:

$$
i \mathcal{T}^{\sigma}=i \Gamma^{\sigma q q} D_{\sigma}(p) i \Gamma^{\sigma \pi \pi}
$$

where $D_{\sigma}$ is the meson propagator. As it was noticed above, the meson propagator in the NJL model has the form (5), but in the $1 / N_{c}$ consideration at $T=0$ it is reasonable to consider the meson propagator in the pole approximation:

$$
D_{M}(x)=\frac{g_{M q q}^{2}}{M_{M}^{2}-x-i \Gamma_{M} M_{M}},
$$

where $M_{M}$ is the meson mass, $g_{M q q}$ is the meson-quark coupling constant and $\Gamma_{M}$ is the meson width.

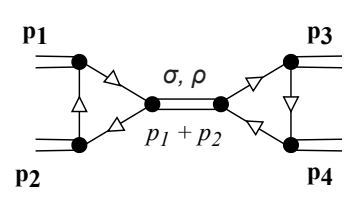

d)

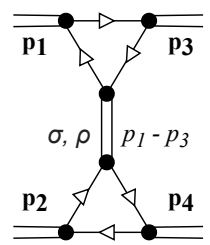

e)

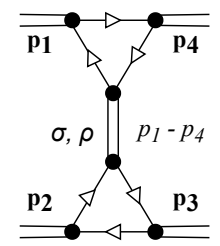

f)

Figure 3. The meson-exchange diagrams for $\pi \pi$ scattering

The $\sigma \rightarrow \pi \pi$ triangle amplitude is written as:

$$
i \Gamma^{\sigma \pi \pi}=-g_{\sigma \pi \pi} g_{\pi q q}^{2} \mu^{2 \epsilon} \int \frac{d^{d} k}{(2 \pi)^{d}} \frac{\operatorname{Tr}\left\{\Gamma_{1}\left(\hat{q_{1}}+m\right) \Gamma_{2}\left(\hat{q_{2}}+m\right) \Gamma_{3}\left(\hat{q_{3}}+m\right)\right\}}{\left[q_{1}^{2}-m^{2}\right]\left[q_{2}^{2}-m^{2}\right]\left[q_{3}^{2}-m^{2}\right]},
$$

where vertex factors $\Gamma_{i}$ are $\Gamma_{i}=\Gamma_{\pi}=i \gamma_{5} g_{\pi q q}$ for pion and $\Gamma_{i}=\Gamma_{\sigma}=\mathbf{1} g_{\sigma q q}$ for $\sigma$-meson, the momenta $q_{i}$ depend on the diagram.

$\operatorname{In}[14]:=$

triags $=$ LoopIntegrate[Spur $\left[\gamma \cdot \mathrm{k}+m_{1}, \gamma_{5}, \gamma \cdot\left(\mathrm{k}-p_{2}\right)+m_{1}, \mathbf{1}, \gamma \cdot\left(\mathrm{k}+p_{1}\right)+m_{1}, \gamma_{5}\right], \mathrm{k}$ $\left.\mathrm{k}, \mathrm{m},\left(\mathrm{k}-p_{2}\right), \mathrm{m}, \mathrm{k}+p_{1}, \mathrm{~m}\right]$;

triagu $=$ LoopIntegrate[Spur[ $\left[\gamma \cdot \mathrm{k}+m_{1}, \gamma_{5}, \gamma \cdot\left(\mathrm{k}-p_{3}\right)+m_{1}, \mathbf{1}, \gamma \cdot\left(\mathrm{k}-p_{1}\right)+m_{1}, \gamma_{5}\right], \mathrm{k}$, $\left.\mathrm{k}, \mathrm{m},\left(\mathrm{k}-p_{3}\right), \mathrm{m}, \mathrm{k}-p_{1}, \mathrm{~m}\right]$;

triagt $=$ LoopIntegrate[Spur[ $\left.\gamma \cdot \mathrm{k}+m_{1}, \gamma_{5} \gamma \cdot\left(\mathrm{k}-p_{4}\right)+m_{1}, \mathbf{1}, \gamma \cdot\left(\mathrm{k}-p_{1}\right)+m_{1}, \gamma_{5}\right], \mathrm{k}, \mathrm{k}$, $\left.\mathrm{m},\left(\mathrm{k}-p_{4}\right), \mathrm{m}, \mathrm{k}-p_{1}, \mathrm{~m}\right]$;

$\operatorname{In}[17]:=$

sTriagInt $=$ LoopRefine[triags $/$. onShell]

uTriaglInt = LoopRefine[triagt $/$. onShell];

tTriagInt $=$ LoopRefine[triagu $/$. onShell];

Out[17]:=

$-8 \mathrm{~m}-4 \mathrm{~m} \operatorname{DiscB}[\mathrm{s}, \mathrm{m}, \mathrm{m}]-4 \mathrm{~m}\left(\frac{1}{\epsilon}+\log \left[\frac{2}{\mathrm{~m}^{2}}\right]\right)+2 \mathrm{~m}\left(2 \mathrm{M}^{2}-\mathrm{s}\right) \operatorname{ScalarC0}\left[\mathrm{M}^{2}, \mathrm{M}^{2}, \mathrm{~s}, \mathrm{~m}, \mathrm{~m}\right.$, $\mathrm{m}]$ 
Combining the result for triangle diagrams and the propagator (9) according to equation (8), the final result for the meson-exchange diagram can be obtained. The real and imaginary parts of the amplitudes as a function $s$ are shown in Figure 4 for the case $t=0$. It is clearly seen in Figures 2, 4 that all results at $t=0$ have the threshold at $s \sim M_{\sigma}$.
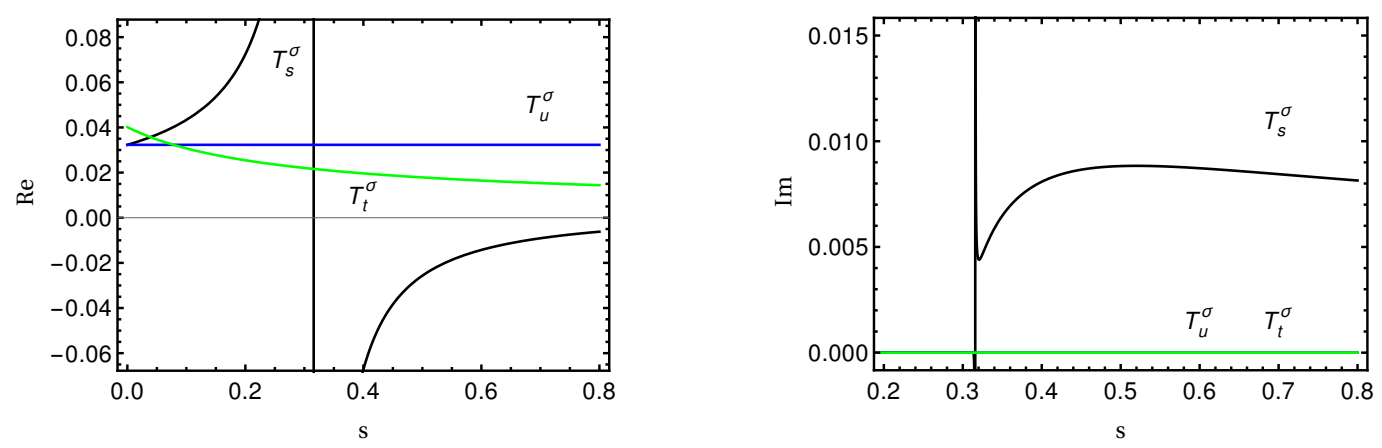

Figure 4. The real (left panel) and imaginary (right panel) parts of the sigma-exchange $\pi \pi$ scattering amplitudes for the case $t=0$

\section{The scattering lengths and the cross section}

The invariant scattering amplitude in common view has the form [14]:

$$
\begin{aligned}
& \left\langle c p_{c} ; d p_{d}|\mathcal{T}| a p_{a} ; b p_{b}\right\rangle= \\
& \quad=\delta_{a b} \delta_{c d} A(s, t, u)+\delta_{a c} \delta_{b d} B(s, t, u)+\delta_{a d} \delta_{b c} C(s, t, u)
\end{aligned}
$$

where $s, t, u$ are the Mandelstam variables and $s+t+u=4 M_{\pi}^{2}$ for the $\pi \pi$-scattering. The amplitude of definite total isospin $I(I=0,1,2)$ defined by $A_{I}$, can be projected out from equation (11):

$$
A_{0}=3 A+B+C, \quad A_{1}=B-C, \quad A_{2}=B+C .
$$

The functions $A(s, t, u), B(s, t, u), C(s, t, u)$ are combined taking into account the isospin factors in the amplitudes in equations (7) (see Table 2).

Some practical results can be obtained after combining the functions $A(s, t, u), B(s, t, u), C(s, t, u)$ and restoring factors $\left(g_{\pi q q}^{4}\right)$ in integrals $\mathcal{T}_{s, t, u}^{b o x}, \mathcal{T}_{s, t, u}^{\sigma}$. When the scattering is at the kinematic threshold, the scattering lengths can be obtained as $a_{i}=\frac{1}{32 \pi} A_{i}$. At the conditions $s=4 M_{\pi}^{2}, u=t=0$, the length $a_{1}=0$ as the $a_{1}$ has the meaning of the scattering "volume" [15].

In this work the parameters from the equations (6) were used with $M_{\pi}=$ $0.14, M_{\sigma}=0.562, m=0.28, g_{\pi q q}=3.66, g_{\sigma q q}=2.66$. For these parameters the values $a_{0}=0.147 a_{2}=-0.0475$ were obtained.

As the general kinematics was used to write the amplitude $\mathcal{T}$, the total cross section can be evaluated. According to the charge and isospin conservation law, the following reaction for $\pi \pi$-scattering can exist: $\pi^{0} \pi^{0} \rightarrow \pi^{0} \pi^{0}, \pi^{0} \pi^{0} \rightarrow \pi^{+} \pi^{-}$, 
$\pi^{ \pm} \pi^{ \pm} \rightarrow \pi^{ \pm} \pi^{ \pm}, \pi^{+} \pi^{-} \rightarrow \pi^{+} \pi^{-}$. In this work for analysis the reaction $\pi^{+} \pi^{-} \rightarrow \pi^{+} \pi^{-}$was chosen as the most complete set of experimental data can be found for this reaction. The total amplitude for this case with taking into account the Clebsch-Gordan coefficients and isospin amplitudes is:

$$
\mathcal{T}^{\pi^{+} \pi^{-} \rightarrow \pi^{+} \pi^{-}}=\frac{1}{6} A_{2}+\frac{1}{2} A_{1}+\frac{1}{3} A_{0} .
$$

Table 2

The isospin factors

\begin{tabular}{|c|c|}
\hline Diagram & Isospin factor \\
\hline $\mathcal{T}_{s}^{b o x}$ & $2\left(\delta^{a b} \delta^{c d}+\delta^{a c} \delta^{b d}-\delta^{a d} \delta^{c b}\right)$ \\
$\mathcal{T}_{t}^{b o x}$ & $2\left(\delta^{a b} \delta^{c d}-\delta^{a c} \delta^{b d}+\delta^{a d} \delta^{c b}\right)$ \\
$\mathcal{T}_{u}^{b o x}$ & $2\left(-\delta^{a b} \delta^{c d}+\delta^{a c} \delta^{b d}+\delta^{a d} \delta^{c b}\right)$ \\
$\mathcal{T}_{s}^{\sigma}$ & $4 \delta^{a b} \delta^{c d}$ \\
$\mathcal{T}_{t}^{\sigma}$ & $4 \delta^{a c} \delta^{b d}$ \\
$\mathcal{T}_{u}^{\sigma}$ & $4 \delta^{a d} \delta^{c b}$ \\
\hline
\end{tabular}

In Figure 5 (left panel) the real, imaginary parts and the absolute value of the scattering amplitude are shown for the case $t=0$.
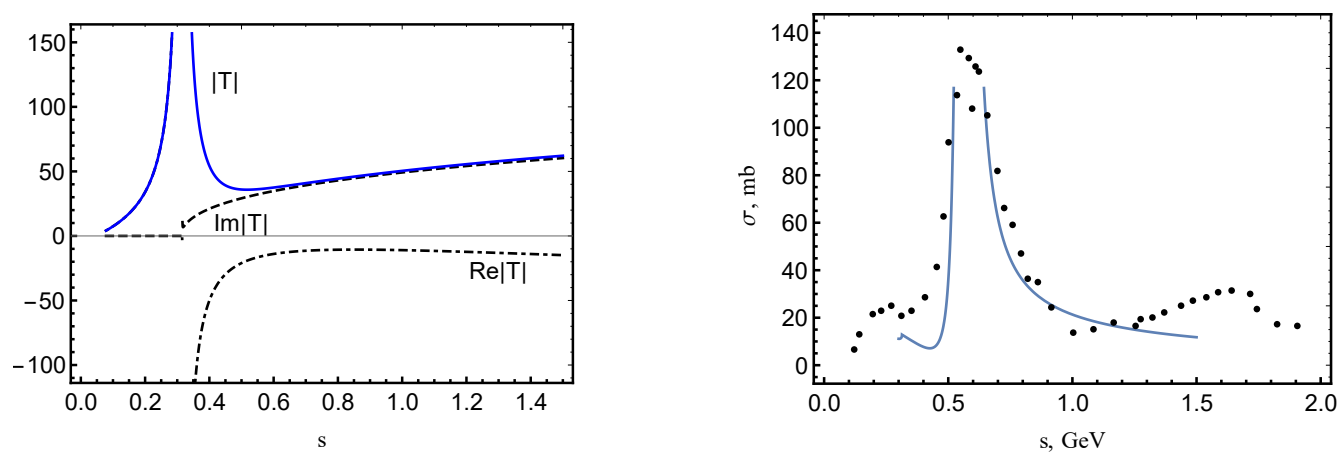

Figure 5. Left panel: the real, imaginary parts and absolute value for the scattering amplitude for the reaction $\pi^{+} \pi^{-} \rightarrow \pi^{+} \pi^{-}$at $t=0$. Right panel: total cross section

The total cross section in the center of mass of the system is defined using the standard expression:

$$
\sigma_{\mathrm{el}}=\frac{1}{16 \pi \lambda\left(s, m^{2}\right)} \int_{t-}^{t^{+}} d t\left|\mathcal{T}^{\pi^{+} \pi^{-} \rightarrow \pi^{+} \pi^{-}}\right|^{2}
$$


where $\lambda\left(s, M_{\pi}^{2}\right)=\left(s-4 M_{\pi}^{2}\right) s, t^{+}=0$ and $t^{-}=4 M_{\pi}^{2}-s$. It is clearly seen, that in the center of mass of the system there exists the kinematic threshold for the reaction: $s>4 M_{\pi}^{2}$.

In literature there is a wide range for the $\sigma$-meson mass and its width $M_{\sigma}(400 \div 1000), \Gamma_{\sigma}(600 \div 1000)$ can be found. In Fig. 5 (right panel) the total cross section for the reaction $\pi^{+} \pi^{-} \rightarrow \pi^{+} \pi^{-}$is shown with experimental data taken from [16], [17]. The result was obtained for $M_{\sigma}=0.75 \mathrm{GeV}$, $\Gamma_{\sigma}=0.05 \mathrm{GeV}, g_{\pi q q}=4$.

\section{Conclusion}

The pions are involved in many physical processes in a wide energy range. For instance, during the heavy ion collision the pions are the final state for many hadronic decays and moreover, the pion annihilation contributes to the dilepton spectra. The electroweak pion decays $\left(\pi^{ \pm} \rightarrow \mu^{ \pm}+\nu_{\mu}\right.$ or $\left.\pi^{0} \rightarrow 2 \gamma\right)$ are involved into a star cooling, etc. That is why the pion pion scattering attracts the special interest.

In this work the $\pi \pi$-scattering amplitude is calculated in the frame $\mathrm{SU}(2)$ NJL model based on the QCD Lagrangian. The model reproduces the most important low-energy theorems, describes the mechanism of spontaneous chiral symmetry breaking, vector dominance, mass spectra for light mesons and strong and electroweak decays of the ground meson states. When to the model the Polyakov-loop dynamics is added, which as a classical field couples to quarks, the model describes the confinement properties [18]. The main advantage of the NJL-like models is the possibility to include the temperature as a parameter using the Matsubara mechanism, that makes the model applicable to the description of matter under critical condition.

Nevertheless, the calculation of the pion-pion scattering amplitude within the framework of this model meets difficulties when the general kinematics and finite temperature are used (see [1], [2], [12]). Nowadays the finite temperature amplitude was obtained in the kinematic limit $s=4 M_{\pi}^{2}, u=t=0$. This limit leads to the correct values of the scattering lengths $a_{0}, a_{1}, a_{2}$, but does not allows to calculate the total cross section, lifetime or width of the pion. Other models, for example, sigma-model gives the simple expression for the scattering amplitude [19], but does not take into account the quark structure of mesons.

In this work the pion-pion scattering amplitude was obtained in the frame of the NJL model at $T=0$ using the dimensional regularization of the integrals, which is used in Package-X [9]. To make a simple check of the program, the scattering lengths $a_{0}$ and $a_{2}$ were obtained. At zero temperature, the results are $a_{0}=0.147, a_{2}=-0.0475$, which are consistent with the Weinberg values $a_{0}^{W}=7 M_{\pi}^{2} / 32 \pi f_{\pi}^{2}=0.158, a_{1}^{W}=M_{\pi}^{2} / 24 \pi f_{\pi}^{2}=0.03 a_{2}^{W}=$ $-2 M_{\pi}^{2} / 32 \pi f_{\pi}^{2}=-0.045$ [20] and other NJL results [2], [12]. At the threshold $s=4 M_{\pi}^{2}, u=t=0$ the length $a_{1}=0$, as it plays the role of a volume of the reaction. Besides that, the most important contribution to $a_{1}$ is made by the diagrams with $\rho$-meson, which is not included in the model with Lagrangian (1). 
The total cross section for the reaction $\pi^{+} \pi^{-} \rightarrow \pi^{+} \pi^{-}$is shown in Figure 5 with experimental data from [16], [17]. The difference in experimental and model results is due to the fact that the simplest model with pseudo-scalar and scalar meson was used. The scattering amplitude involved only "box"and $\sigma$ - exchange diagrams, skipping more high-mass mesons. At the same time for model the $M_{\sigma} \sim 0.7 \mathrm{GeV}$ was used instead of $0.4-0.55 \mathrm{GeV}$ according to PDG. It is obviously that including the vector $\rho$-meson with the mass $\sim 0.77 \mathrm{GeV}$ would lead to the shift of the maximum to the correct point with $s \sim 0.7 \mathrm{GeV}$. The axial-vector $a_{1}$-meson with higher mass is a reason for the second maximum at $s>1.5 \mathrm{GeV}$. To take into account $\rho$-meson and other heavy mesons, the Lagrangian (1) should be changed and the set of equations (6) should be self-consistently extended for additional mesons.

\section{Acknowledgments}

This work was supported by the Russian Fund for Basic Research (RFBR) under grant no. 18-02-40137.

\section{References}

[1] S. P. Klevansky, "The Nambu-Jona-Lasinio model of quantum chromodynamics," Reviews of Modern Physics, vol. 64, pp. 649-708, 3 Jul. 1992. DOI: $10.1103 /$ RevModPhys .64.649.

[2] Wei-jie Fu and $\mathrm{Yu}$-xin Liu, "Mesonic excitations and pi-pi scattering lengths at finite temperature in the two-flavor Polyakov-Nambu-JonaLasinio model," 2009. arXiv: 0904.2914 [hep-ph].

[3] V. Jos. (1989). "FORM (symbolic manipulation system)," [Online]. Available: https://www.nikhef .nl/ form/.

[4] P. Kasper. (2020). "Cadabra," [Online]. Available: https://cadabra. science/.

[5] R. Mertig, M. Bohm, and A. Denner, "FEYN CALC: Computer algebraic calculation of Feynman amplitudes," Computer Physics Communications, vol. 64, pp. 345-359, 1991. DOI: 10.1016/0010-4655(91)90130-D.

[6] T. Hahn and M. Perez-Victoria, "Automatized one loop calculations in four-dimensions and D-dimensions," Computer Physics Communications, vol. 118, pp. 153-165, 1999. DOI: 10.1016/S0010-4655(98) 00173-8. arXiv: hep-ph/9807565.

[7] T. Binoth, J.-P. Guillet, G. Heinrich, E. Pilon, and T. Reiter, "Golem95: A Numerical program to calculate one-loop tensor integrals with up to six external legs," Computer Physics Communications, vol. 180, pp. 23172330, 2009. DOI: $10.1016 / \mathrm{j} . \mathrm{cpc} .2009 .06 .024$. arXiv: 0810.0992 [hep-ph].

[8] G. Passarino and M. Veltman, "One-loop corrections for $e^{+} e^{-}$annihilation into $\mu^{+} \mu^{-}$in the Weinberg model," Nuclear Physics B, vol. 160, no. 1, pp. 151-207, 1979. DOI: 10.1016/0550-3213(79)90234-7. 
[9] H. H. Patel, "Package-X: A Mathematica package for the analytic calculation of one-loop integrals," Computer Physics Communications, vol. 197, pp. 276-290, Dec. 2015. DOI: 10.1016/j .cpc.2015.08.017.

[10] D. Ebert, Y. L. Kalinovsky, L. Munchow, and M. K. Volkov, "Mesons and diquarks in a NJL model at finite temperature and chemical potential," International Journal of Modern Physics A, vol. 8, pp. 1295-1312, 1993. DOI: $10.1142 / \mathrm{S} 0217751 \mathrm{X} 93000539$.

[11] M. L. Goldberger and S. B. Treiman, "Decay of the pi meson," Physical Review, vol. 110, pp. 1178-1184, 1958. DOI: 10.1103/PhysRev.110. 1178.

[12] E.Quack, P. Zhuang, Y. L. Kalinovsky, S. P. Klevansky, and J.Hufner, " $\pi \pi$ scattering lengths at finite temperature," Physics Letters B, vol. 348, pp. 1-6, 1995.

[13] S. Narison, "Techniques of dimensional regularization and the two-point functions of QCD and QED," Physics Reports, vol. 84, no. 4, pp. 263399, 1982. DOI: 10.1016/0370-1573(82)90023-0.

[14] J. D. Bjorken and S. D. Drell, Relativistic quantum mechanics, ser. International series in pure and applied physics. New York, NY: McGraw-Hill, 1964.

[15] H. J. Schulze, "Pion pion scattering lengths in the SU(2) Nambu-JonaLasinio model," Journal of Physics G, vol. 21, pp. 185-191, 1995. DOI: $10.1088 / 0954-3899 / 21 / 2 / 006$.

[16] V. Srinivasan et al., " $\pi^{-} \pi^{+} \rightarrow \pi^{-} \pi^{+}$interactions below $0.7 \mathrm{GeV}$ from $\pi^{-} p \rightarrow \pi^{-} \pi^{+} n$ data at $5 \mathrm{GeV} / \mathrm{c}, "$ Physical Review D, vol. 12, pp. 681692, 3 Aug. 1975. DOI: 10.1103/PhysRevD.12.681.

[17] S. D. Protopopescu, M. Alston-Garnjost, A. Barbaro-Galtieri, S. M. Flatté, J. H. Friedman, T. A. Lasinski, G. R. Lynch, M. S. Rabin, and F. T. Solmitz, " $\pi \pi$ Partial-Wave Analysis from Reactions $\pi^{+} p \rightarrow$ $\pi^{+} \pi^{-} \Delta^{++}$and $\pi^{+} p \rightarrow K^{+} K^{-} \Delta^{++}$at $7.1 \mathrm{GeV} / \mathrm{c}, "$ PPhysical Review D, vol. 7, pp. 1279-1309, 5 Mar. 1973. DOI: 10.1103/PhysRevD.7.1279.

[18] Y. L. Kalinovsky, V. D. Toneev, and A. V. Friesen, "Phase diagram of baryon matter in the $\mathrm{SU}(2) \mathrm{Nambu}$ - Jona-Lasinio model with a Polyakov loop," Physics-Uspekhi, vol. 59, no. 4, pp. 367-382, 2016. DOI: $10.3367 /$ UFNe.0186.201604b.0387.

[19] S. R. Cotanch and P. Maris, "QCD based quark description of pi pi scattering up to the sigma and rho region," Physical Review D, vol. 66, p. 116010,2002 . DOI: $10.1103 /$ PhysRevD . 66 .116010. arXiv: hep$\mathrm{ph} / 0210151$.

[20] V. Bernard, U. G. Meissner, A. Blin, and B. Hiller, "Four point functions in quark flavor dynamics: Meson meson scattering," Physics Letters B, vol. 253, pp. 443-450, 1991. DOI: 10.1016/0370-2693 (91)91749-L.

\section{For citation:}

Y. L. Kalinovsky, A. V. Friesen, E. D. Rogozhina, L. I. Golyatkina, Application of a computer algebra systems to the calculation of the $\pi \pi$-scattering 
amplitude, Discrete and Continuous Models and Applied Computational Science 28 (3) (2020) 216-229. DOI: 10.22363/2658-4670-2020-28-3-216-229.

Information about the authors:

Friesen, Alexandra V. - Candidate of Physical and Mathematical Sciences, researcher of Joint Institute for Nuclear Research (e-mail: avfriesen@theor.jinr.ru, phone: +79645528418, ORCID: https://orcid.org/0000-0002-6744-6766)

Rogozhina, Elizaveta D. - Student of Dubna State University; Senior laboratory assistant of Joint Institute for Nuclear Research, (e-mail: liorinoff@mail.ru, phone: +79017790706, ORCID: https://orcid.org/0000-0002-5408-6749)

Golyatkina, Lyubov' I. - Student of Dubna State University, Senior laboratory assistant of Joint Institute for Nuclear Research, (e-mail: lubovgolyatkina@mail.ru, phone: 890178950, ORCID: https://orcid.org/0000-0002-6549-0341)

Kalinovsky, Yuriy L. - Doctor of Physical and Mathematical Sciences, senior researcher of Joint Institute for Nuclear Research (e-mail: kalinov@jinr. ru, phone: +79653257685, ORCID: https://orcid.org/0000-0002-7596-5531) 
DOI: $10.22363 / 2658-4670-2020-28-3-216-229$

\title{
Применение средств компьютерной алгебры к вычислению амплитуды $\pi \pi$-рассеяния
}

\author{
Ю. Л. Калиновский ${ }^{1,2}$, А. В. Фризен ${ }^{1}$, Е. Д. Рогожина ${ }^{1,2}$, \\ Л. И. Голяткина ${ }^{1,2}$ \\ ${ }^{1}$ Объединённый институт ядерных исследований \\ ул. Жолио-Кюри, д. 6, Дубна, Московская область, 141980, Россия \\ 2 Университет «Дубна» \\ ул. Университетскал, д. 19, Дубна, Московская область, 141982, Россия
}

Целью данной работы является разработка программ для расчёта амплитуды рассеяния элементарных частиц, а также автоматизация таких расчётов с использованием систем компьютерной алгебры (Mathematica, Form, Cadabra). В статье рассматривается процесс рассеяния пиона на пионе в рамках эффективной КХД-мотивированной модели Намбу-Иона-Лазинио с двумя ароматами кварков. Для расчёта амплитуды рассеяния (начиная с расчёта Фейнмановских диаграмм и заканчивая вычислением Фейнмановских интегралов в однопетлевом приближении) использовался пакет Package-X для Mathematica. Интегралы Фейнмана в однопетлевом приближении вычислялись для случая общей кинематики. В Package-X в основе вычисления интегралов лежит метод Фейнмановской параметризации с последующей пространственной регуляризацией. Для проверки корректности вычислений был произведён расчёт длин рассеяния $a_{0}=0,147$ и $a_{2}=-0,0475$ для случая нулевой температуры, и было построено полное сечение рассеяния как функции $s$. Полученные результаты сравнивались с другими моделями и экспериментальными данными.

Ключевые слова: Фейнмановские интегралы, однопетлевое приближение, полное сечение рассеяния, длины рассеяния, системы компьютерной алгебры, Package-X 